https://helda.helsinki.fi

Pain assessment in native and non-native language : difficulties in reporting the affective dimensions of pain

\title{
Mustajoki, Marianne
}

2018-10

Mustajoki , M , Forsen , T \& Kauppila , T 2018 , ' Pain assessment in native and non-native language : difficulties in reporting the affective dimensions of pain ' , Scandinavian journal of pain , vol. 18 , no. 4 , pp. 575-580 . https://doi.org/10.1515/sjpain-2018-0043

http://hdl.handle.net/10138/321658

https://doi.org/10.1515/sjpain-2018-0043

cc_by_nc_nd

publishedVersion

Downloaded from Helda, University of Helsinki institutional repository.

This is an electronic reprint of the original article.

This reprint may differ from the original in pagination and typographic detail.

Please cite the original version. 


\section{Clinical pain research}

Marianne Mustajoki*, Tom Forsén and Timo Kauppila

\section{Pain assessment in native and non-native language: difficulties in reporting the affective dimensions of pain}

https://doi.org/10.1515/sjpain-2018-0043

Received February 27, 2018; revised May 7, 2018; accepted May 11, 2018; previously published online July 10, 2018

\begin{abstract}
Background and aims: The language in assessing intensity or quality of pain has been studied but the results have been inconsistent. The physicians' language skills might affect the estimation of the severity of pain possibly leading to insufficient use of analgesics. Several interfering cultural factors have complicated studies aimed at exploring the language used to detect the quality of pain. We aimed to compare native and non-native language related qualitative aspects of pain chosen by Swedish speaking patients with diabetes.

Methods: In the study participated 10 Finnish and 51 Swedish speaking patients with diabetes. The Pain Detect-questionnaire was used for clarifying the patients' pain and the mechanism of their pain (neuropathic or not) and for assessing the intensity and quality of pain. In addition, the patients completed the short-form McGill Pain Questionnaire (sfMPQ) in Finnish (test I). After 30 min the subjects completed the SfMPQ a second time in their native language (test II). The Swedish speakers estimated their second language, Finnish, proficiency on a 5-graded scale.

Results: There were significantly more discrepancies between sfMPQ test I and test II among the Swedish speaking respondents who reported poor (hardly none) Finnish language proficiency compared with those with good Finnish proficiency. Discrepancies occurred especially between the affective qualities of pain.
\end{abstract}

\footnotetext{
*Corresponding author: Marianne Mustajoki, University of Helsinki, Department of General Practice and Primary Health Care, Kiskontie 23 B, 00280 Helsinki, Finland, Phone +358 503810837 , E-mail:marianne.mustajoki@duodecim.fi

Tom Forsén and Timo Kauppila: University of Helsinki, Department of General Practice and Primary Health Care, Helsinki, Finland
}

Conclusions: Poor second language proficiency exposes Swedish speakers to pain communication difficulties related to the affective aspects of pain. Consequently, discordant language communication could cause underestimation of the severity of pain and pain undertreatment. Implications: To ensure adequate pain treatment measuring the affective dimension of pain in the patient's native language is crucial.

Keywords: pain; communication; language proficiency; diabetes; bilingual; minority.

\section{Introduction}

A mutual language usually improving communication and understanding is critical in generating good and functional relationships between healthcare personnel and patients. Language barriers can cause communication difficulties which may compromise the diagnosis and treatment of a disease [1]. The physicians' language skills might affect the estimation of pain, resulting in poorer diagnostic confidence and increased need of ancillary tests [2]. This may also lead to insufficient and nonoptimal use of analgesics [3-5]. Measuring pain intensity in a clinical setting is per se challenging and can easily be underestimated by the healthcare personnel [6-8].

Measuring pain is a multifaceted process and consists of observing both the intensity and the quality of pain [9-11]. The role of language in assessing the pain intensity and quality has been studied but the results have been inconsistent [3-5, 12]. Spanish speaking cancer patients in the USA reported a lower level of pain if they had estimated the general practitioner's (GP) language skills good in the patient-preferred language [13].

The importance and impact of language in mediating qualitative pain related information has not been studied because of several interfering factors, including cultural ones, hampering measurements. Finland is by tradition an ethnically and culturally broadly homologous but 
bilingual country where patients have the right to get healthcare services either in Finnish or Swedish. However, the language transition in the Swedish speaking community from Swedish to Finnish has accelerated during the last decades [14].

We aimed to investigate language related qualitative aspects of pain with the McGill Pain Questionnaire (MPQ) validated in Finnish and Swedish [15-17]. The qualitative aspects of pain can be studied with the short-form, sfMPQ, developed for detection and measurement of both sensory and affective qualities of pain [15]. The SfMPQ has been widely used in clinical pain studies and validated in several languages [18]. Diabetes is associated with pain, commonly due to neuropathy. We studied to what extent the self-rated ability to use the second or weaker national language, in bilingual subjects diagnosed with diabetes compromises measurement of qualitative aspects of pain with sfMPQ. We specifically studied the results of the pain questionnaire in two languages in order to identify how dependent the expression of both the sensory and affective dimensions of pain were on the language used by the patient.

\section{Materials and methods}

\subsection{Subjects}

Sixty-one Finnish and Swedish speaking diabetic patients aged 18-68 years participated in the study. The study participants were sampled from one healthcare center in South Ostrobothnia and one in the metropolitan area as well as from the Finnish Diabetes Association. The data was collected during the years 2013-2016.

The characteristics of the study participants were recorded according to their age, gender, weight, height, educational attainment, marital status, occupation, duration of type I and II diabetes and native language. The Swedish speakers were separately instructed to estimate their proficiency in Finnish on a 5-graded scale: 0 (hardly at all), 1 (some ability to speak Finnish), 2 (moderate, e.g. fair ability to speak Finnish), 3 (good, e.g. Finnish almost as good as mother language Swedish) and 4 (Finnish as good as mother language Swedish).

\subsection{Setting and measurement tools}

The respondents were instructed to complete the validated Pain Detect-questionnaire in their native language [19]. The Pain Detect questionnaire was used to clarify systematically the patients' pain and the mechanism of their pain (neuropathic or not), and furthermore, to evaluate the intensity of pain with a numerical pain rating scale (0-10) [19]. Secondly all respondents completed the sfMPQ in Finnish (test I). After 30 min the Finnish speaking respondents repeated the SfMPQ in Finnish (test II) to reveal intrinsic repetition variations. The Swedish speakers repeated the SfMPQ test (test II) in their native language, Swedish [17].

\subsection{Main outcome measurement}

The SfMPQ includes fourteen questions about the quality of the experienced pain. The adjectives chosen by the patient in the two tests with SfMPQ were compared with each other. Choosing the same adjective to describe the pain in the same question in test I and test II was scored zero (0). Choosing a different adjective in test II the particular question was scored one (1) indicating discrepancy in the qualitative estimate. Thus, each patient could have a score discrepancy between 0 and 14 .

The first 10 questions of sfMPQ reflect sensory and the questions from 11 to 14 reflect affective dimensions of pain [15-17]. Analogously to the former, the patients could have sensory discrepancy scores between 0 and 10 and on the affective dimension scores between 0 and 4 .

Our study was approved by the Ethics Committee of the Hospital District of Helsinki and Uusimaa (379/E0/06). All the study participants provided the written informed consent.

\subsection{Statistical analysis}

The Swedish speaking participants differentiated in four Finnish language proficiency groups were compared with each other and with the Finnish speaking group with parametric ANOVA followed by Bonferroni-test, nonparametric ANOVA with Dunns' test or with $\mathrm{X}^{2}$-test, when appropriate. The statistical significance was set at $p<0.05$.

\section{Results}

The questionnaire was completed by 51 Swedish speaking and 10 Finnish speaking persons diagnosed with diabetes. One third of the Swedish speaking participants reported their Finnish language proficiency close to their native language. Nine subjects reported "hardly any" or "some" Finnish proficiency and were pooled to the group 
Table 1: Educational status of the respondents.

\begin{tabular}{|c|c|c|c|c|c|}
\hline Basic education & $\begin{array}{r}\text { Finnish speaking } \\
\text { respondents }\end{array}$ & $\begin{array}{r}\text { Finnish as good } \\
\text { as Swedish }\end{array}$ & $\begin{array}{r}\text { Good Finnish } \\
\text { proficiency }\end{array}$ & $\begin{array}{r}\text { Moderate Finnish } \\
\text { proficiency }\end{array}$ & $\begin{array}{r}\text { Poor Finnish } \\
\text { proficiency }\end{array}$ \\
\hline Elementary school & 0 & 2 & 2 & 3 & 6 \\
\hline Studying & 0 & 0 & 2 & 1 & 0 \\
\hline Academic degree & 0 & 1 & 4 & 2 & 1 \\
\hline Vocational degree & 10 & 11 & 9 & 5 & 2 \\
\hline
\end{tabular}

"poor” Finnish language proficiency. Fourteen reported "good" and 11 "moderate" Finnish language proficiency. The respondents were predominantly females $(75 \%)$. The Swedish speaking group did not differ significantly from the comparison group regarding age, gender or duration of diabetes ( $p$-values $>0.05$ ). Educational attainment varied somewhat within the Swedish speaking group. Those who reported poor Finnish proficiency were less educated and had mostly completed only compulsory schooling, whereas those who spoke Finnish as their mother tongue had at least a basic level of vocational education ( $\mathrm{X}^{2}$-test, $p<0.05$, Table 1). The occupational status did not differ significantly between the Finnish and Swedish speaking participants ( $p$-value $>0.05$ ) (Supplementary Material).

No significant differences in the intensity of selfreported pain were observed between the Finnish speaking group and the Swedish speaking groups, $8\{4.5\}$ [median and inter quartile range, IQR \{\}$]$ in the Finnish speaking group, $5\{5\}$ in the group "Finnish as good as Swedish" $5\{5.5\}$ in the group "good Finnish proficiency", $5\{8\}$ in the group "moderate Finnish proficiency" and $7\{5.5\}$ in the group "poor Finnish proficiency". Self-reported body mass index $\left(\mathrm{BMI} \mathrm{kg} / \mathrm{m}^{2}\right)$ did not differ between the groups.

There were significantly more discrepancies between sfMPQ test I and test II among the Swedish speaking respondents who reported poor (hardly any and fair) Finnish language proficiency compared to those with good Finnish proficiency (ANOVA, $p<0.001$ ) (Fig. 1). The comparison between the Finnish and the Swedish speaking participants did not reveal any differences in the sensory qualities of pain with sfMPQ (Fig. 2A). However, the testre-test results showed that the Swedish speaking participants reporting only poor Finnish language proficiency demonstrated significantly more discrepancies between the affective qualities of pain than the Finnish speaking participants ( $p<0.01$, non-parametric ANOVA) (Fig. 2B).

PainDETECT scores suggested that five Finnish speaking participants suffered from pain likely to be of neuropathic origin. Two of the Swedish speakers who described their Finnish language proficiency as "as good as their mother language" reported pain that was possibly of neuropathic origin. Three Swedish speakers with "good", two

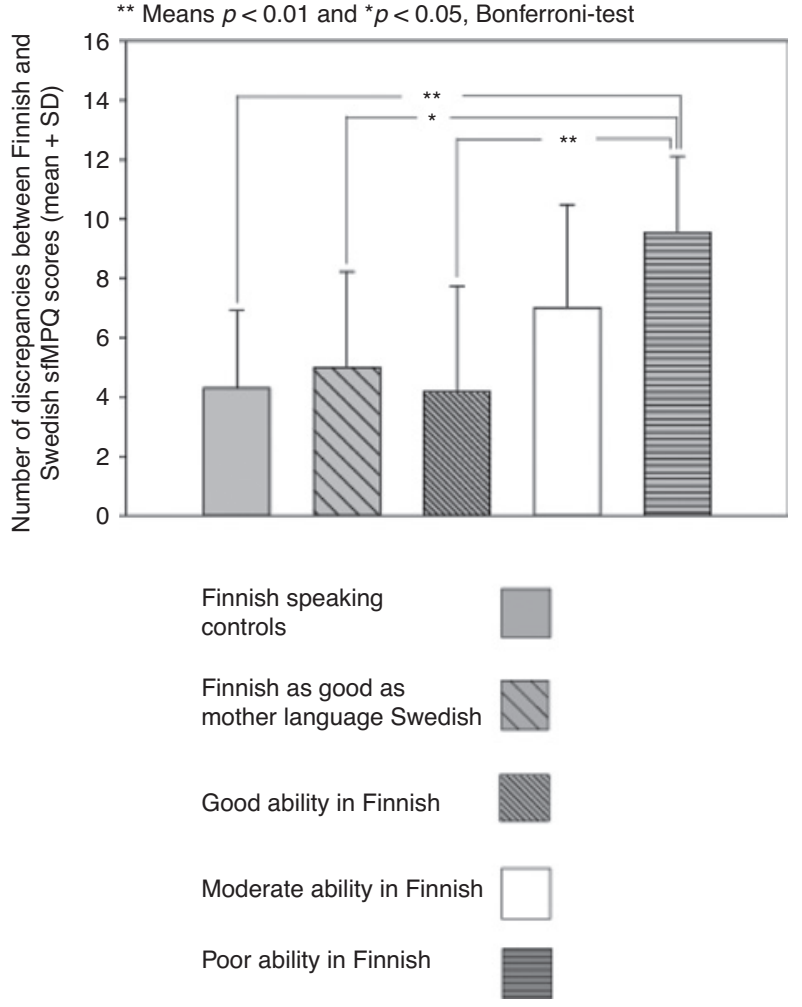

Fig. 1: Discrepancies between the Finnish and Swedish sfMPQ scores related to the second language proficiency.

with "moderate" and four with "poor" Finnish proficiency also reported pain possibly of neuropathic origin.

\section{Discussion}

The Swedish speaking participants with diabetes reporting poor Finnish proficiency chose significantly more often another quality of pain description compared to the choice in their native language. The descriptions of particularly the affective quality of pain in either native or nonnative language varied significantly among the Swedish speakers reporting poor Finnish language proficiency. No similar differences were observed in their description of the sensory aspects of pain. Swedish speakers with poor 


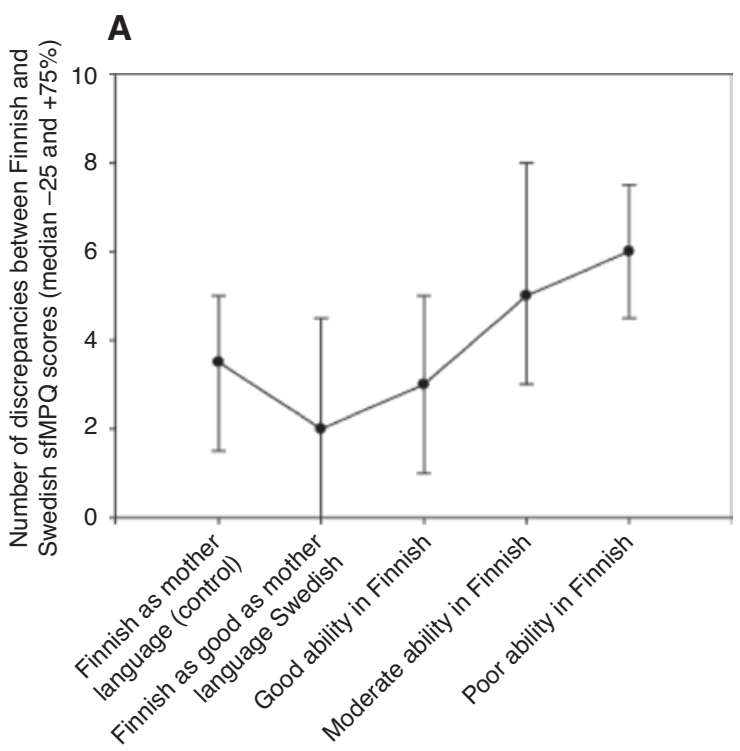

B

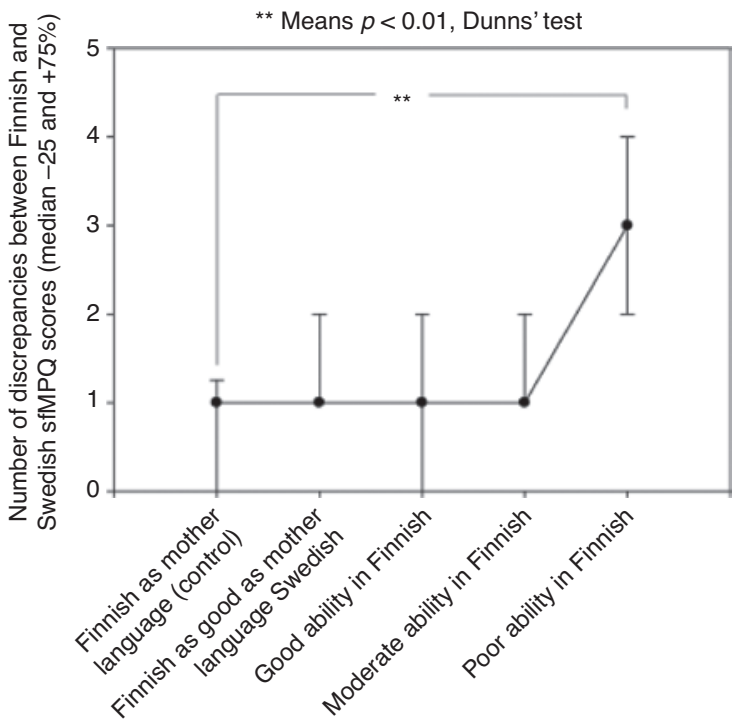

Fig. 2: Discrepancies between the Finnish and Swedish sensory sfMPQ scores (A) and affective scores (B) related to the second language proficiency.

Finnish language proficiency were less educated than the Finnish speaking participants. Compared to the native Finnish speaking participants, the Swedish speakers reporting moderate or good Finnish language proficiency did not differ in any of the studied aspect.

There are two major qualitative compartments in pain to measure, namely the sensory-discriminative and sensory affective-motivational components [20-22]. Whereas the sensory discriminative component tells more about the intensity and location of pain, the affective-motivational component tells about the meaning of the pain to the individual and is more closely related to the behaviour the pain triggers [23]. We did not separately observe signs of anxiety or stress among the Swedish speakers with poor second language proficiency, although anxiety sensitivity might contribute to the affective interpretations of somatic sensations [24]. Our present finding suggests that poor second language proficiency exposes Swedish speakers to communication difficulties with the Finnish speaking healthcare providers. The Swedish speakers may have difficulties in finding words to describe affective aspects of pain, possibly providing a partial explanation for the findings that pain treatment is insufficient among minorities [3-5].

How could the observed difficulties of the patients with poor language concordance be explained and what might cause them? The connotation of words in the native and second language, especially those related to emotions, has been reported to differ among bilingual individuals [25]. Our findings demonstrated that this phenomenon also concerns culturally homologous and substantially bilingual minorities unquoted in any previous studies.

The language has impact on the comprehension of the pain descriptors derived from MPQ and all of them are perhaps not words used by the Swedish speakers for explaining pain experience in real life [26]. Yet the situation during the study, e.g. unhurried multiple-choice task when choosing the correct adjective to describe the quality of pain, is hardly as difficult and prone to misunderstanding as the real, verbally mediated clinical situation during a busy healthcare visit. Furthermore, the results of those reporting better Finnish proficiency than poor suggest that both Finnish and Swedish speakers use equally often different everyday words for describing pain. These interferences cannot therefore explain the differences between the choices of the quality of pain description.

Discordant language communication might cause underestimation of the severity of pain resulting in undertreatment of their symptom, as also observed in previous studies [1]. Our findings support the importance of communicating about the affective dimension of pain in the patients' native language.

The language minorities' generally poorer health conditions and utilisation of healthcare services have been verified in several studies [27, 28]. However, the Swedish speaking minority Finns' health conditions differ from these, showing a more favourable health [29]. Our study demonstrated that conceiving bilingual, socially wellintegrated minority patients as one homogeneous group seems inconsistent with the real clinical situation. Those with poor second language proficiency may have a double 
burden when facing the healthcare system: they are devoid of common native language with the healthcare personnel and they have less personal socioeconomic prerequisites. This interesting association found in our study requires further research.

We revealed that the Swedish speakers are generally bilingual and able to sufficiently comprehend words in their second language. However, an unknown number of them seem to have a weaker initial communication position which influences their pain experience negatively. The individual differentiations of bilingualism represent many language proficiency variations which are challenging to identify during a short medical interview. When aiming to measure pain exactly and correctly by interviewing, there is a strong need for communicating in a concordant language with all patients.

The weakness of the study was the relatively small sample. By testing more Swedish speaking patients the results could have provided stronger evidence. Furthermore the SfMPQ test in Swedish and Finnish consist considerable many artificial words not in use in everyday life among patients. Poor Finnish proficient and less educated Swedish speakers' language difficulties in both Swedish and Finnish might have the increased differences between the language groups.

\section{Limitations}

We implemented our study in the region where the Swedish speaking population is mainly residing. The study proved to be challenging due to the difficulty in patient recruiting caused by the relatively time-consuming test. The prolonged data collecting time has however, not distorted the results as changes in the Swedish speakers' second language proficiency seem very slow.

\section{Authors' statements}

Research funding: This study was supported by The Swedish Cultural Foundation in Finland.

Conflict of interest: We have no conflict of interest to declare. All authors have seen and approved the manuscript being submitted. We warrant that the article is an original work. We certify that the article has not received prior publication and is not under consideration for publication elsewhere.

Informed consent: All the study participants provided the written informed consent approved by the Ethics Committee of the Hospital District of Helsinki and Uusimaa (379/ E0/06).
Ethical approval: Our study was approved by the Ethics Committee of the Hospital District of Helsinki and Uusimaa (379/E0/06).

\section{References}

[1] Cioffi J. Communicating with culturally and linguistically diverse patients in an acute care setting: nurses' experiences. Int J Nurs Stud 2003;40:299-306.

[2] Garra G, Albino H, Chapman H, Singer AJ, Thode HC. The impact of communication barriers on diagnostic confidence and ancillary testing in the emergency department. J Emerg Med 2010;5:681-5.

[3] Cleeland CS. Pain and treatment of pain in minority patients with cancer. Ann Intern Med 1997;127:813-6.

[4] McNeill JA, Sherwood GD, Starck PL, Nieto B. Pain management outcomes for hospitalized Hispanic patients. Pain Manag Nurs 2001;2:25-36.

[5] McCaffery M, Pasero C. Culturally sensitive pain control. Am J Nurs 1999;99:18-20.

[6] Puntillo K, Neighbor M, O’Neil N, Nixon R. Accuracy of emergency nurses in assessment of patients' pain. Pain Manag Nurs 2003;4:171-5.

[7] Davoudi N, Afsharzadeh P, Mohammadalizadeh S, Haghdoost AA. A comparison of patients' and nurses' assessments of pain intensity in patients with coronary artery disease. Int J Nurs Pract 2008;14:347-56.

[8] Melotti RM, Samolsky Dekel BG, Carosi F, Ricchi E, Chiari P, D’Andrea R, Di Nino G. Categories of congruence between inpatient self-reported pain and nurses' evaluation. Eur J Pain 2009;13:992-1000.

[9] Bond MR, Pilowsky I. Subjective assessment of pain and its relationship to the administration of analgesics in patients with advanced cancer. J Psychosom Res 1966;10:203-8.

[10] Melzack R. The McGill Pain Questionnaire: major properties and scoring methods. Pain 1975;1:277-99.

[11] Dubuisson D, Melzack R. Classification of clinical pain descriptions by multiple group discriminant analysis. Exp Neurol 1976;51:480-7.

[12] Todd KH, Lee T, Hoffman JR. The effect of ethnicity on physician estimates of pain severity in patients with isolated extremity trauma. JAMA 1994;271:925-8.

[13] Mosher CE, Duhamel KN, Egert J, Smith MY. Self-efficacy for coping with cancer in a multiethnic sample of breast cancer patients: associations with barriers to pain management and distress. Clin J Pain 2010;26:227-34.

[14] Finnäs F. Finlandssvenskarna 2009. En statistisk rapport. 2010. The Swedish Assembly of Finland. p. 23, figure 10. h www. folktinget.fi.

[15] Melzack R. The short-form McGill Pain Questionnaire. Pain 1987;30:191-7.

[16] Ketovuori H, Pöntinen PJA. Pain vocabulary in Finnish - the Finnish pain questionnaire. Pain 1981:11:247-53.

[17] Burckhardt CS, Bjelle A. A Swedish version of the short-form McGill Pain Questionnaire. Scand J Rheumatol 1994;23:77-81.

[18] Hawker GA, Mian S, Kendzerska T, French M. Measures of adult pain: Visual Analog Scale for Pain (VAS Pain), Numeric Rating 
Scale for Pain (NRS Pain), McGill Pain Questionnaire (MPQ), Short-Form McGill Pain Questionnaire (SF-MPQ), Chronic Pain Grade Scale (CPGS), Short Form-36 Bodily Pain Scale (SF-36 BPS), and Measure of Intermittent and Constant Osteoarthritis Pain (ICOAP). Arthritis Care Res (Hoboken) 2011;63 Suppl 11:S240-52.

[19] Freynhagen R, Baron R, Gockel U, Tölle TR. PainDETECT: a new screening questionnaire to identify neuropathic components in patients with back pain. Curr Med Res Opin 2006;22:1911-20.

[20] Gracely RH, McGrath P, Dubner R. Validity and sensitivity of ratio scales of sensory and affective verbal pain descriptors: manipulation of affect by diazepam. Pain 1978;5:19-29.

[21] Gracely RH, McGrath P, Dubner R. Narcotic analgesia: fentanyl reduces the intensity but not the unpleasantness of painful tooth pulp sensations. Science 1979;203:1261-3.

[22] Price DD. Psychological and neural mechanisms of the affective dimension of pain. Science 2000;288:1769-72.

[23] Auvray M, Myin E, Spence C. The sensory-discriminative and affective-motivational aspects of pain. Neurosci Biobehav Rev 2010;34:214-23.

[24] Schroeder S, Gerlach AL, Martin A. Implicit affective evaluation of somatosensory sensations in patients with non-cardiac chest pain. J Behav Ther Exp Psychiatry 2014;45:381-8.
[25] Itzak I, Vingron N, Baum SR, Titone D. Bilingualism in the real world: how proficiency, emotion and personality in a second language impact communication in clinical and legal setting. Transl Issues Psychol Sci 2017;3:48-65.

[26] Wilson D, Williams M, Butler D. Language and the pain experience. Physiother Res Int 2009;14:56-65.

[27] Schenker Y, Karter AJ, Schillinger D, Warton EM, Adler NE, Moffet HH, Ahmed AT, Fernandez A. The impact of limited English proficiency and physician language concordance on reports of clinical interactions among patients with diabetes. Patient Educ Couns 2010;81:222-8.

[28] Díaz E, Miskemen T, Vega WA, Gara M, Wilson DR, Lesser I, Escamilla M, Neighbors HW, Arndt S, Strakowski S. Inconsistencies in diagnosis and symptoms among bilingual and English-speaking Latinos and Euro-Americans. Psychiatr Serv 2009;60:1379-82.

[29] Nieminen T, Prättälä R, Martelin T, Hyyppä MT, Alanen E, Koskinen S. Social capital, health behaviors and health: a population based associational study. BMC Public Health 2013;13:613.

Supplementary Material: The online version of this article offers supplementary material (https://doi.org/10.1515/sjpain-2018-0043). 